\title{
Overview of the AO calibration strategies in the ELT context
}

\author{
Cedric T. Heritier ${ }^{\mathrm{a}, \mathrm{b}, \mathrm{c}, \mathrm{d}}$, Thierry Fusco ${ }^{\mathrm{b}}$, Benoit Neichel ${ }^{\mathrm{a}}$, Simone Esposito ${ }^{\mathrm{c}}$, Sylvain Oberti ${ }^{\mathrm{d}}$, \\ Carlos Correia ${ }^{a}$, Jean-François Sauvage ${ }^{b}$, Charlotte Bond ${ }^{\mathrm{e}}$, Olivier Fauvarque ${ }^{\mathrm{a}}$, Enrico Pinna ${ }^{\mathrm{c}}$, \\ Guido Agapito ${ }^{\mathrm{c}}$, Alfio Puglisi ${ }^{\mathrm{c}}$, Johann Kolb ${ }^{\mathrm{d}}$, Pierre-Yves Madec ${ }^{\mathrm{d}}$, and Clémentine Bechet ${ }^{\mathrm{f}}$ \\ a Aix Marseille Université, CNRS, LAM (Laboratoire d'Astrophysique de Marseille) UMR \\ 7326, 13388, Marseille, France

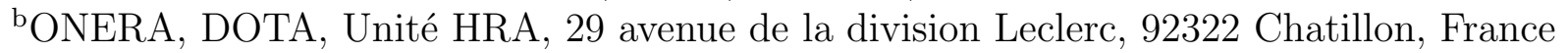 \\ ${ }^{\mathrm{C}} \mathrm{INAF}$ - Osservatorio Astrofisico di Arcetri Largo E. Fermi 5, 50125 Firenze, Italy \\ ${ }^{d}$ European Southern Observatory, Karl-Schwarzschild-str-2, 85748 Garching, Germany \\ eInstitute for Astronomy,University of Hawaii,640 N. Aohoku Place,Hilo,HI 96720

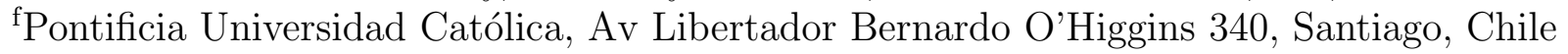

\begin{abstract}
The scientific potential of the ELT will rely on the performance of its AO systems that will require to be perfectly calibrated before and during the operations. The actual design of the ELT will provide a constraining environment for the calibration and new strategies have to be developed to overcome these constraints. This will be particularly true concerning the Interaction Matrix of the system with no calibration source upward M4 and moving elements in the telescope. After a brief presentation of the ELT specificities for the calibration, this communication focuses on the different strategies that have already been developed to get/measure the Interaction Matrix of the system, either based on synthetic models or using on-sky measurements. First tests of these methods have been done using numerical simulations for a simple AO system and a proposition for a calibration strategy of the ELT will be presented.
\end{abstract}

Keywords: Adaptive Optics, Calibration, Interaction Matrix, ELT, On-Sky Interaction Matrix, Pseudo-Synthetic Interaction Matrix

\section{INTRODUCTION}

Within a decade, the new generation of Extremely Large Telescopes (ELT) will make a breakthrough in the ground based Astronomy. The scientific potential of these giants relies on challenging new Adaptive Optics (AO) systems, integrated inside the telescope itself, and providing images to all the instrumentation downstream. The complexity of these instruments leads to new problematics, especially concerning the calibration of the Interaction Matrix (IM), the link between the Wave Front Sensor (WFS) measurements and the Deformable Mirror (DM) actuators. The registration between the WFS and the DM may evolve dynamically with the telescope environment, making the calibration detuned, and resulting in a loss of the AO system performance. These telescopes will also provide a new and constraining environment with no direct access to intermediate focal plane in front of the DM and thus no external calibration source.

In this context, accelerating the calibration procedures or performing it on sky, if possible during the AO correction itself, becomes necessary. ${ }^{1}$ Some strategies have already been developed and are currently tested/validated on current $8 \mathrm{~m}$ telescope facilities such as the $\mathrm{AOF}^{2,3}$ at the VLT and $\mathrm{FLAO}^{4-6}$ at the LBT. A first idea consists in computing a theoretical model of the IM and update it during the operation by identifying key parameters, either on-sky or directly using AO closed-loop data. ${ }^{7-9}$ A second idea is to acquire the IM directly on-sky. In this communication, we focus on some of these calibration strategies to evaluate their feasibility on an ELT, putting light on their drawbacks, advantages or limits and offering eventual optimization.

\footnotetext{
Further author information: E-mail: cedric.heritier@lam.fr/ heritier@arcetri.astro.it/cheritie@eso.org
} 


\section{CONTEXT}

\subsection{Calibration of an AO system}

The good behavior of any AO system relies on two main calibration phases: the construction of its Interaction Matrix (IM) and the calibration of the Non Common Path Aberrations (NCPA). Figure 1a illustrates where these calibrations phases take place in a classical AO system.

To build the IM, the most common way consists in poking on each actuator of the DM (or apply a modal basis for a modal IM) and record the corresponding WFS signals. By inverting this matrix, one gets a reconstruction matrix (often called "Reconstructor") that is used to compute the commands to apply on the DM for a given WFS measurement. This experimental process ensures to take into account any misregistration lying between the DM and the WFS. The term misregistration stands here for any shift, rotation of the DM actuators with respect to the WFS subapertures or any higher order of pupil distortion.

The accuracy of the IM is crucial as the system is extremely sensitive to any drift in the calibration (especially for high order systems) and it will consistently impact the AO system performance. Defining the quality of such an object is then not easy to do (SNR? Conditioning number? Eigenvalues spectrum?) as it mostly depends on the characteristics of the system. So far, the best way to evaluate the quality of an IM is to try using it in closed loop and study the effectiveness of the correction.

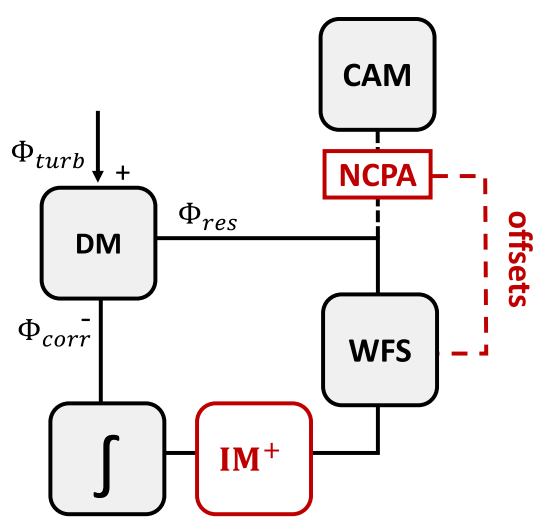

(a) Calibration of an $\mathrm{AO}$ system.

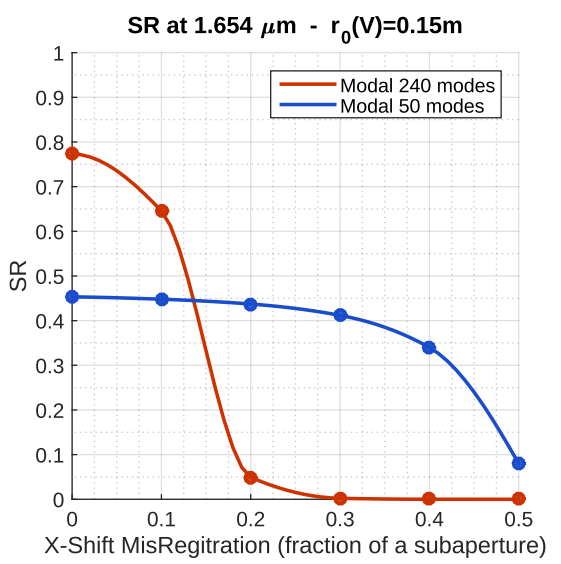

(b) Impact of a shift mis-registration on the performance.

Figure 1: Calibration of an AO System (a) and (b) sensibility to an horizontal shift for two IM controlling 50 and 240 modes.

The sensibility to misregistrations depends mostly on the geometry of the DM but also on the number of modes controlled in the IM. Figure 1b shows the impact of a shift of the DM actuators for a classical AO system defined in Table 1. This plot shows that a high order system provides better AO performance but will be extremely sensitive to any misregistration while a low-order system maintains its performance despite high misregistration values. Therefore, a trade-off has to be made on the number of modes to control and the sensibility to misregistrations.

\subsection{AO calibration in the ELT context}

In the ELT case, the situation will be completely different as the DM will be located in the telescope itself, few tens of meters away from the WFS so that the registration between the WFS and the DM may evolve dynamically with the telescope. This specificity already provides a new constraining environment as it will require frequent updates of the IM, and this even during the operations. Moreover, there won't be any direct access to an intermediate focal plane in front of the DM, and thus no external calibration source to calibrate initially the system. 
The number of actuators of the DM will drastically increase (around 5000 actuators), increasing the calibration time. The DM will also have a different geometry than the Fried's one with 6 petals composed of around 860 actuators and organised in bricks. ${ }^{10}$

Therefore, new methods and optimization of the calibration procedures have to be developed accounting for ELT specificities: complex models of both DM and WFS, fast calibration necessary with no or low impact on the operations, large number of actuators and moving elements in the system. So far, different strategies have been identified and remain to be investigated to meet with the ELT requirements or speed up the measurements:

- On-Sky IM: We measure the interaction matrix on-sky, finding a way to get rid of the turbulence effects and speed up the calibration procedures (Fast push-pull?, ${ }^{1}$ using Hadamard matrix to speed up the process? ${ }^{11,12}$ Modulation/Demodulation $\left.{ }^{4-6}\right)$.

- Synthetic IM: We build a model of the AO system and we generate an IM from it. ${ }^{1}$

- Pseudo-Synthetic IM: We build a synthetic IM and we measure some key-parameters on-sky to estimate the registration of the system and update the IM during the operation. ${ }^{2,3,7-9}$

The next sections will focus on some of these new strategies that are being tested on numerical simulations of a classical AO system with Pyramid WFS.

\subsection{Numerical Simulation Tool}

The simulations presented in this paper were done using the OOMAO tool that stands for Object Orient Matlab Adaptive Optics. ${ }^{13}$ The properties of the system defined in these simulations are given in Table 1 but it mostly consists in a Single Conjugate Adaptive Optics (SCAO) system with Natural Guide Star (NGS) for an 8-m class telescope and Pyramid WFS. This reduced size system was chosen to speed up the simulation and simulations on an ELT-size telescope will be done once the best strategy and its implementation will be defined.

\begin{tabular}{|c|c|c|}
\hline \multirow{3}{*}{ Atmosphere } & Wavelength & $0.55 \mu \mathrm{m}$ \\
& $r_{0}$ & $15 \mathrm{~cm}$ \\
& $L_{0}$ & $30 \mathrm{~cm}$ \\
& $\mathrm{Cn}^{2}$ profile & 3 layers \\
\hline \multirow{3}{*}{ Telescope } & Diameter & $8 \mathrm{~m}$ \\
& Central Obstruction & None \\
& Resolution & $128 \mathrm{pix}$ \\
\hline \multirow{2}{*}{ Science } & Wavelength & $\mathrm{H}(1.65 \mu \mathrm{m}$ \\
& Magnitude & 10 \\
\hline \multirow{2}{*}{ NGS } & Wavelength & $\mathrm{I}(0.79 \mu \mathrm{m})$ \\
& Magnitude & 8 \\
\hline
\end{tabular}

\begin{tabular}{|c|c|c|}
\hline \multirow{4}{*}{ WFS } & Subapertures & $16 \times 16$ \\
& Modulation & $3 \lambda / \mathrm{D}$ \\
& Detector RON & $0.1 \mathrm{e}-$ \\
& Detector Photon Noise & On \\
\hline \multirow{4}{*}{ DM } & Controlled Actuators & 241 \\
& Pitch & $0.5 \mathrm{~m}$ \\
& Influence Functions & Gaussian \\
& Mechanical Coupling & $30 \%$ \\
\hline \multirow{2}{*}{ Loop } & Frequency & $500 \mathrm{~Hz}$ \\
& Delay & 2 frames \\
& Nominal Performance & $70 \% \mathrm{SR}$ \\
\hline
\end{tabular}

Table 1: Numerical Simulations Parameters

The modal basis used here consists in 150 Karhunen-Loève modes generated from Zernikes polynomial, diagonalizing the turbulence covariance matrix of the Zernikes. ${ }^{14}$ The linearity and sensibility plots to these modes are given in Figure 2 with the residual PSD of the loop for the mode 1.

\section{ON-SKY INTERACTION MATRIX}

\subsection{Description of the method}

The on-sky calibration faces one major challenge: the impact of the turbulence on the WFS measurements. One way to get rid of it consists in using a periodic modulation signal of the modal basis applied on the DM that is then demodulated in the Fourier space. This method allows even to calibrate several modes at the same time to reduce the calibration time.

A crucial point is that the periodic signals must be injected in a closed loop providing already a good correction such as the sum of the injected signals and the phase residuals remain in the linearity range of the WFS. To do so, as illustrated in Figure 3, the loop is closed using the best reconstructor R available for the system (inverting 


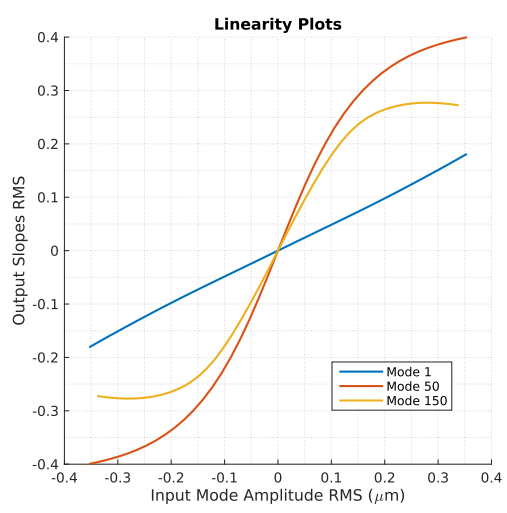

(a) Linearity curves for the modes 1, 50 and 150.

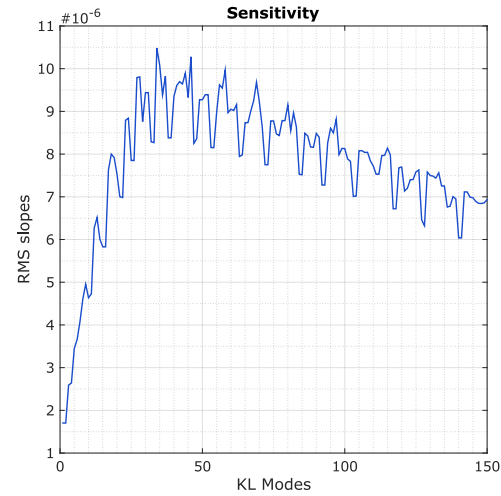

(b) Sensitivity of the PWFS to the KL modes (RMS of the IM columns)

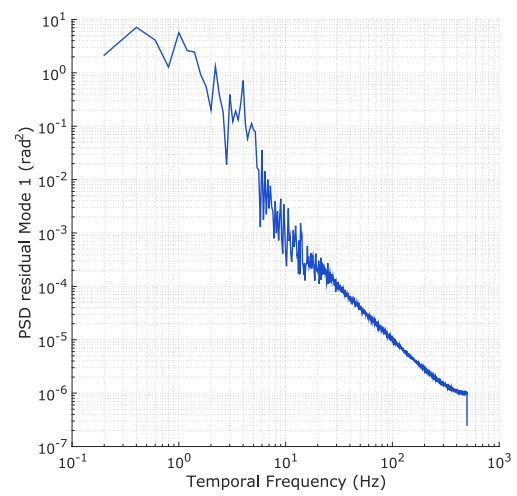

(c) Power Spectrum Density (PSD) of the residual phase for the mode 1

Figure 2: Linearity, sensitivity of the WFS to the KL Modes and residual turbulence spectrum

the $\mathrm{IM}_{l a b}$ that would be measured in lab using an optical calibration source) to be in the optimal conditions (lowest residuals and best correction of the modes introduced). Once the correction is sufficient and the residuals low enough we start modulating the modes:

- One mode $\mathbf{m}$ is modulated at a given frequency (or several modes at several frequencies) using a periodic signal on the DM, the corresponding slopes and command are saved to be sent to a demodulation tool.

- A first demodulation process is achieved on the commands signals (or directly on the DM positions if available) to identify the phase delay $\Phi$ of the signal ( due to the integration time and the command computation) and the amplitude $a_{0}$ of the corresponding mode seen by the WFS. The amplitude injected could indeed be different from the one seen by the WFS.

- A second demodulation is achieved on each pixel of the slopes maps to retrieve the module value of each pixel and its initial sign (phase $\delta$ or $\delta+\pi$ ) providing the slopes vector $s_{0}$ corresponding to the actuation of the mode $\mathbf{m}$.

- Each column of the IM is built by normalizing the slopes vector $s_{0}$ with the amplitude $a_{0}$.

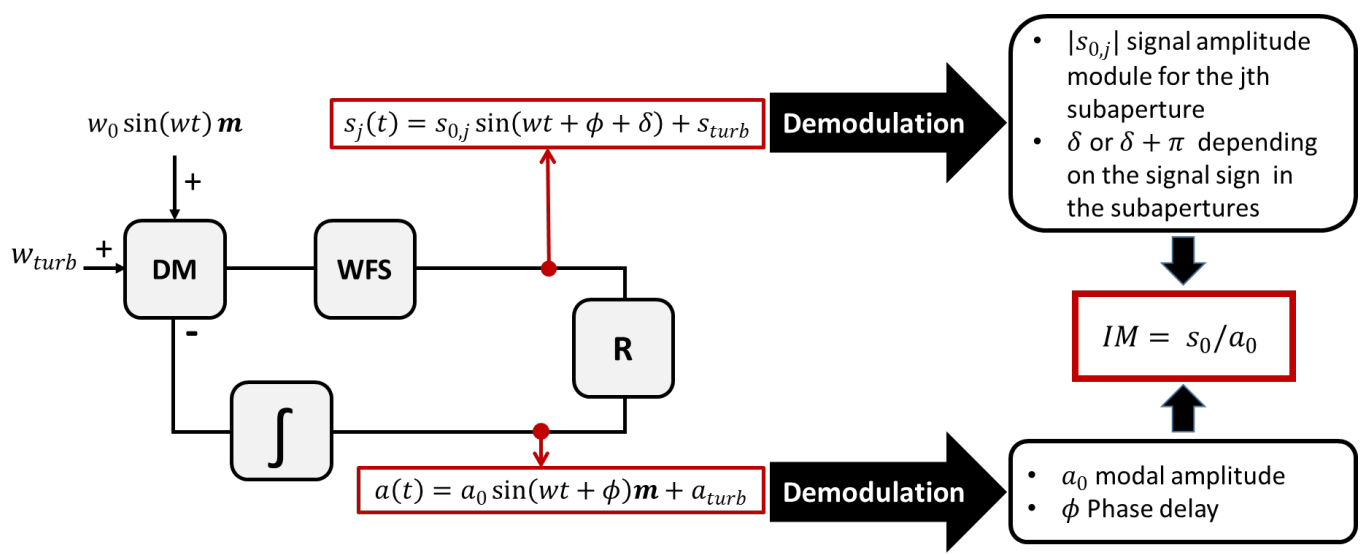

Figure 3: On-Sky IM measurement using periodic signals. 


\subsection{Trade-off study for the parameters}

The goal is to get the best SNR with the lowest impact on science and for the shortest calibration time. Therefore, a trade-off study has to be done for the parameters of the modulation signal. The current study follows an analysis that was done at the LBT. ${ }^{6}$

- Amplitude: If the goal is to record the IM during the observations, the amplitude of the injected signal must be as small as possible to minimize the effects on the operations but large enough to ensure a good SNR of the measurements, without saturating the WFS. Moreover, for a Pyramid WFS, as the sensibility and the linearity is not the same for all the modes, it is necessary to adapt the modes amplitude and flatten the sensibility curve of the WFS (see Figure 2).

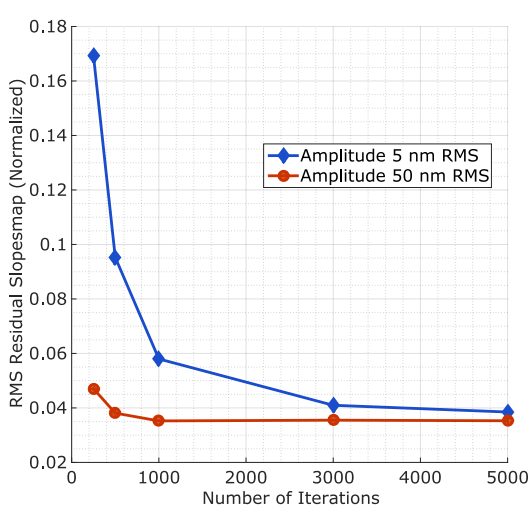

(a) Error on the Slopes Maps retrieved on-sky for different lengths of modulation signals. Results are given for two amplitudes, $5 \mathrm{~nm}$ RMS and 50 nm RMS.

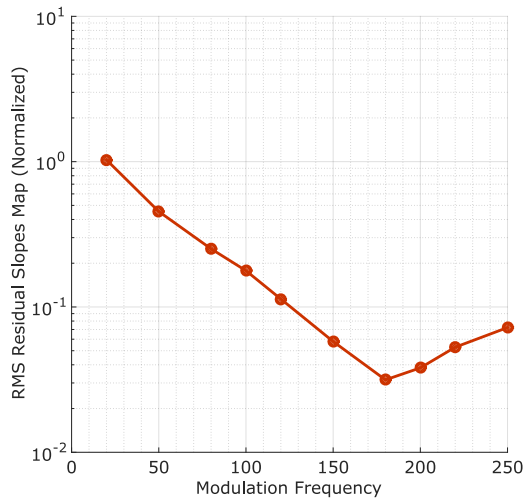

(b) Error on the Slopes Maps retrieved on-sky for different modulation frequency. Results are given for 500 iterations.

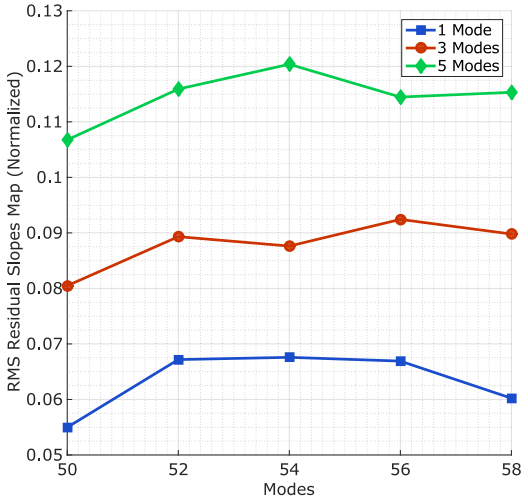

(c) Error on the Slopes Maps retrieved on-sky when modulating 1 mode $(5 \times 500$ iterations $), 3$ modes $(3 \times 500$ iterations $)$ and $5 \operatorname{modes}(500$ iterations) at the same time. $(5 \mathrm{~nm}$ RMS amplitude)

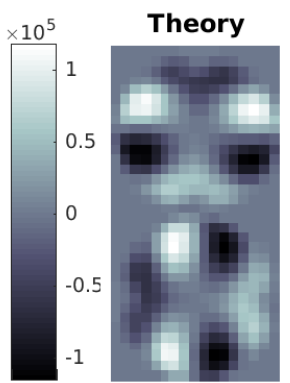

(d) Slopes Map estimation for 250,500,1000,3000 and 5000 iterations with a mode amplitude of $5 \mathrm{~nm}$

Figure 4: Trade-off study for the modulation signal parameters.

Nevertheless, if a dedicated calibration phase (with no observation at the same time) is scheduled to get the IM, the choice for the amplitude can be as large as possible, staying in the linear range of the WFS.

- Sampling The number of measurement points necessary to get a good SNR has to be investigated as the goal is to minimize the calibration time. In our case, we tested several values and we study the quality of the reconstruction for a given mode. The errors on the slopes maps reconstruction are presented in Figure 4a modulating modes at $200 \mathrm{~Hz}$. It seems then necessary to use long modulation signals when applying a small amplitudes but signals can be shorter if using a higher amplitude. 
- Frequency Modulating at high frequencies should give more interesting results as it makes the identification easier in the Fourier spectrum because most of the energy of the turbulence is in the low order frequencies (see Figure 2c). Studying the rejection transfer functions is also an important input because modulating a mode at a frequency that would be either filtered or amplified by the closed loop will disturb the measurements. Morevover, some experimental results ${ }^{6}$ showed the presence of "bad" frequencies and aliasing effects related to other frequencies of the system that need to be investigated ahead of the calibration phase to select a range of "good" frequencies. Figure $4 \mathrm{~b}$ shows that, in our case, the optimal frequency seems to be between 160 and $200 \mathrm{~Hz}$.

- Multiplexing Modulating several signals at different frequencies to lower the calibration time requires to have a perfectly orthogonal basis in the DM space as any cross-talking between the modes will affect the quality of the IM retrieved on-sky. Moreover, there is a price at applying several modes at the same time on the DM as it will have a higher impact the operations. A plot showing the effect of modulating several modes at the same time is given in Figure $4 \mathrm{~d}$ and shows that it also has an impact on the estimation of the slopes maps but allows to reduce the calibration time.

\subsection{Validation of the method}

To validate the method, we apply it for the ideal case: the loop is closed using the $\mathrm{IM}_{l a b}$ that is synthetically measured (no noise) with a system perfectly aligned (no misresgistration). The list of the parameters chosen in this case is given in Table 2 (some of them were chosen to speed up the simulations) and the results displayed in Figure 5.

\begin{tabular}{|c|c|}
\hline Mode Amplitude & $50 \mathrm{~nm}$ RMS \\
\hline Number of iterations/cycle & 500 \\
\hline Multiplexing & 5 modes \\
\hline Misregistrations & None \\
\hline
\end{tabular}

\begin{tabular}{|c|c|}
\hline Number of Modes & 150 \\
\hline Sampling Frequency & $1000 \mathrm{~Hz}$ \\
\hline Modulation frequency & $200-202-204-206-208 \mathrm{~Hz}$ \\
\hline WFS Camera Noise & On \\
\hline
\end{tabular}

Table 2: Modulation Signals Parameters

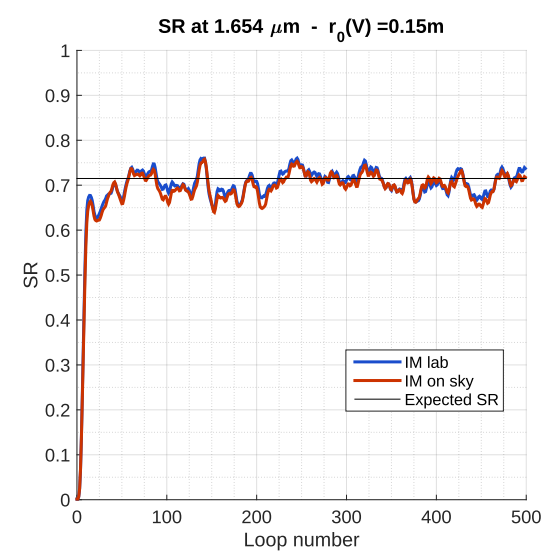

(a) Closing the loop with both $\mathrm{IM}_{\text {on-sky }}$ and $\mathrm{IM}_{l a b}$ for a same turbulent profile

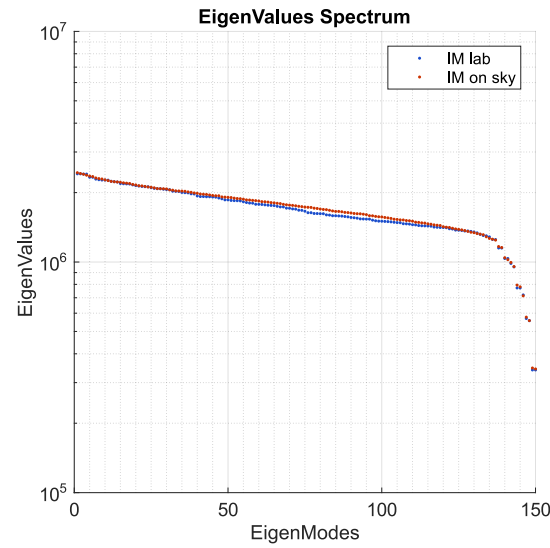

(b) eigenvalues spectrum for both IM: $\mathrm{IM}_{\text {on-sky }}$ and $\mathrm{IM}_{l a b}$

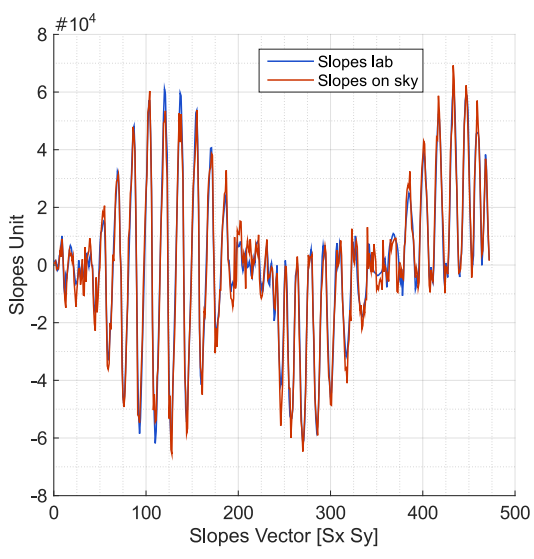

(c) Slopes vector from both IM: $\mathrm{IM}_{\text {on-sky }}$ and $\mathrm{IM}_{l a b}$ (Mode 14)

Figure 5: Validation of the on-sky calibration method using modulation signals

An explicit comparison of both IM, $\mathrm{IM}_{o n-s k y}$ and $\mathrm{IM}_{l a b}$, is given in the plot of Figure 5a: for a same turbulence profile, the corrections are (almost) exactly the same, confirming the good quality of the IM retrieved on sky. 
Figure $5 \mathrm{~b}$ and $5 \mathrm{c}$ give a more detailed comparison with the eigenvalues spectrums and a slopes vector (e.g. one row of the IM concatenating the slopes in X and $\mathrm{Y}$ ). These two plots are also a criteria to confirm the good quality of the $\mathrm{IM}_{\text {onsky }}$ with only small differences that are negligible and will not affect the effectiveness of the corrections.

\subsection{Test of the method with Large Mis-Registrations}

As presented in 2.2, the AO systems of the ELT could be subject to large misregistrations. In that case, can we catch up the nominal performance of the system using the on-sky calibration?

Based on the plot given in Figure 1b, the idea is to apply a static misregistration (X-shift of $25 \%$ of a subaperture) that maintains correct performance (approx. 60\% SR )and then start applying the method. Two IM from the lab are generated:

- $I M_{l a b}^{0}$ : acquired with no noise and when the system was perfectly aligned.

- $I M_{l a b}^{\text {shifted }}$ : acquired with no noise and when the system is shifted of $25 \%$ of a subaperture.

Using the same values for the modulation parameters as in Table 2, the method is applied when the system is shifted. The IM used to closed the loop is $I M_{l a b}^{0}$ which does not correspond to the actual alignment of the system but provides a sufficient correction to start modulating the modes.

The results are presented in Figure 6. We see an improvement in the correction: the $I M_{\text {onsky }}$ catches up pretty well with the performance of the $I M_{\text {lab }}^{\text {shifted }}$ that represents the best IM available for the system (as if the shift was measured before the calibration phase). This shows that if the loop can be closed partially, using an IM that does not fit perfectly with the alignment of the system, it seems possible to apply the method and provide an on-sky IM that takes into account the misregistrations of the system.

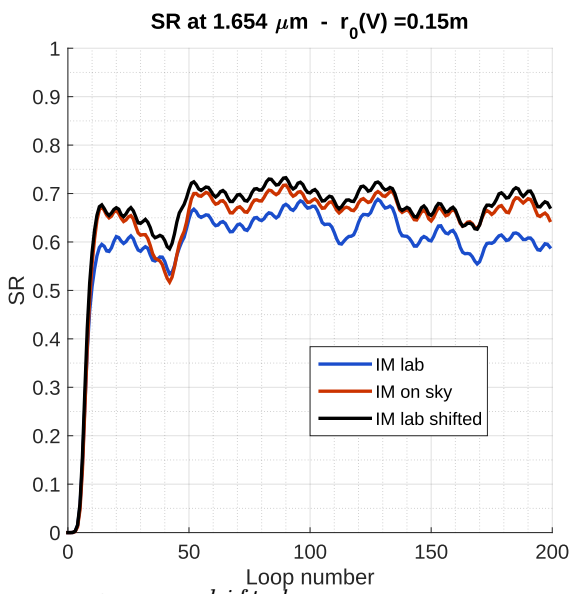

Figure 6: Closing the loop with $I M_{\text {lab }}^{0}, I M_{\text {lab }}^{\text {shifted }}$ and $I M_{\text {onsky }}$ for a same turbulence profile. The

In that case, only 150 modes were retrieved, but an eventual idea would be to close the loop with a low or medium order IM, less sensitive to misregistration (see Figure 1b) and then modulates higher order modes. That way, one could build a high order IM. However, depending on the amplitude, it would maybe require to send commands to the DM to correct the modes injected as the Reconstructor would not "see" the high order modes modulated and would not be able to send the commands to correct it.

\section{PSEUDO-SYNTHETIC INTERACTION MATRIX}

The methods presented here were developed at ESO for the AOF and were based on two ambitious ideas: generate the IM from synthetic models of the system or from closed-loop data to make the AO system free of this calibration phase. 


\subsection{Synthetic or Pseudo-Synthetic Interaction Matrix?}

Theoretically, building a synthetic IM has a lot of advantages: it is noise-free and it can be generated during the day, saving time for the operations. It requires to have perfect models of both WFS and DM, but also that the registration of the system does not evolve between the IM generation and the operation. In some cases, the response of the WFS will also depends on the seeing conditions ( optical gains with a Pyramid,) and are ,therefore, unpredictable. Using only synthetic IM is then doable for an AO system ${ }^{6}$ with really stable alignment but seems complex on a ELT.

What can be done is to generate a synthetic IM from models fed with experimental measurements of misregistration parameters. The IM obtained is then Pseudo-Synthetic (PSIM) and can be easily updated during the operation (accounting for a good estimation of the misregistration parameters during the operations). However, working with synthetic IM, even PSIM, requires to have extremely accurate and sensitive models for both DM and WFS which will not be easy to do for the ELT.

\subsection{Interaction Matrix from closed loop data}

The other idea is to re-compute the IM during the operation using only closed loop data, that would take into account the actual registration of the system. This would have the advantage of tracking the registration state of the system without having any impact on the operation. The method is described in several documents ${ }^{2,8}$ and is illustrated in Figure 7: it requires to have a first IM (measured or synthetic) to invert and close the loop, at least partially, and start saving the interesting signals: DM residual commands and WFS residual slopes.

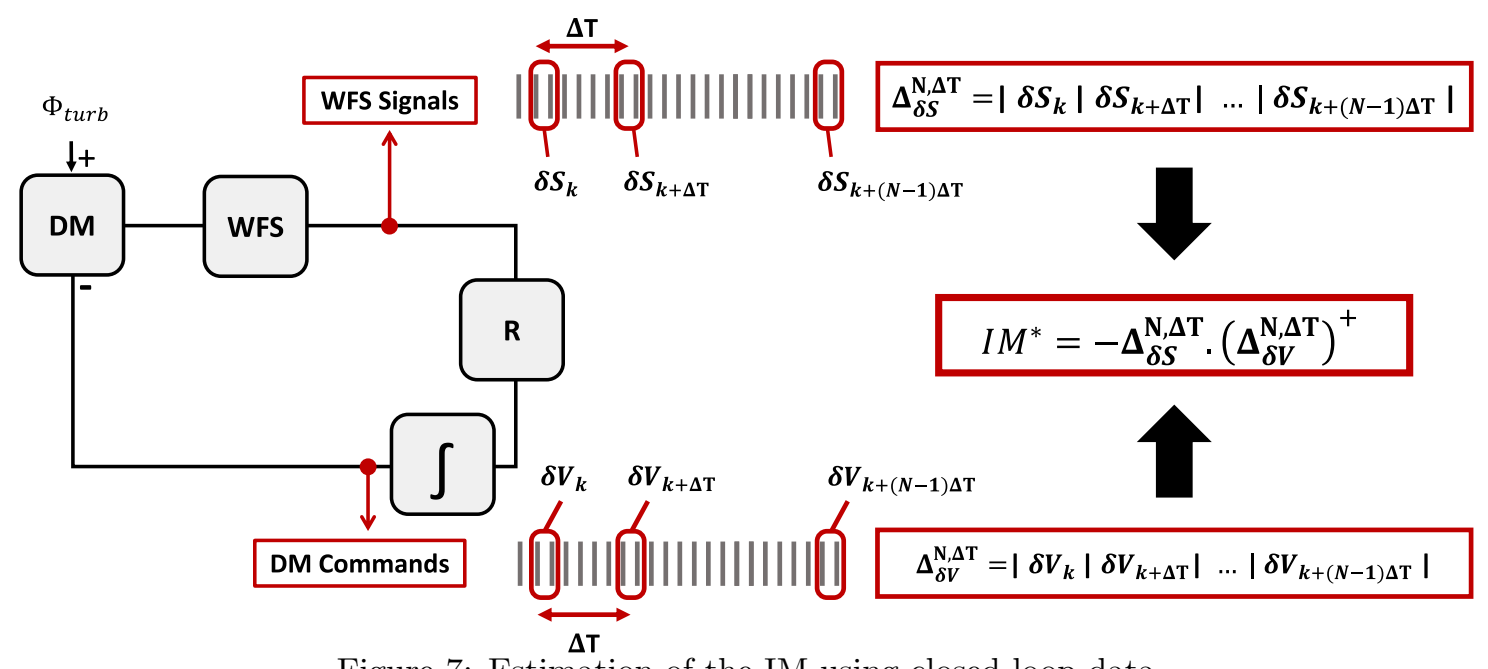

Figure 7: Estimation of the IM using closed loop data

The AO closed-loop equation is:

$$
S_{k}=-I M(p) \cdot V_{k}+M_{W F S} \cdot \Phi_{k}+z_{k}
$$

Where $S$ is the WFS measurement, $V$ the corresponding DM commands, $I M(p)$ the interaction matrix corresponding to the registration parameter $p, M_{W F S}$ the propagation and sensing model, $\Phi$ the turbulence and $z$ the noise associated to the measurement, and $k$ the loop number.

Defining

$$
e_{k}=M_{W F S} \cdot \Phi_{k}+z_{k}
$$

If we consider $\delta S_{k}=S_{k+1}-S_{k}$ :

$$
\delta S_{k}=-I M(p) . \delta V_{k}+\delta e_{k}
$$

If we get rid of $\delta e_{k}$ we can get an estimation of the IM:

$$
I M(p)=-\delta S_{k} / \delta V_{k}
$$


To get rid of the noise, the idea is to average the $\delta e_{k}$ with a large number $N$ of measurements (typically 40000 frames) and de-correlate them by selecting only the $\delta S_{k}$ and $\delta V_{k}$ one every $\Delta T, \Delta T$ being the step parameter that has to be determined to maximize the SNR of the IM studying the correlation of both buffers $\Delta_{\delta S}$ and $\Delta_{\delta V}$ :

$$
\begin{aligned}
& \Delta_{\delta S}^{N, \Delta T}=\left|\delta S_{k}\right| \delta S_{k+\Delta T}|\ldots| \delta S_{k+(N-1) \Delta T} \mid \\
& \Delta_{\delta V}^{N, \Delta T}=\left|\delta V_{k}\right| \delta V_{k+\Delta T}|\ldots| \delta V_{k+(N-1) \Delta T} \mid
\end{aligned}
$$

An estimation of the IM of the system is then:

$$
I M^{*}=-\Delta_{\delta S}^{N, \Delta T} \cdot\left(\Delta_{\delta V}^{N, \Delta T}\right)^{+}
$$

Where ${ }^{+}$stands for the pseudo-inverse. First tests at the $\mathrm{AOF}^{2}$ show that it is indeed possible to retrieve an IM but it is really noisy and has a too low SNR to close the loop. Nevertheless, this noisy IM could provide enough information to estimate some misregistration parameters.(see 4.3)

\subsection{Misregistration identification}

In the frame of this study, the main question is : can we track the registration of the system using only closed loop-data? That way, it would be possible to update the synthetic models during the operations with no impact on science.

One idea to do that is to use the noisy IM retrieved using the closed loop data and project it on a catalog of "sensibility" IM. ${ }^{2}$ This makes the hypothesis that an IM can be expressed as a linear combination of $I M_{0}$ (synthetic for a system perfectly aligned) and sensibility matrix $\delta I M$ (built from the difference between $I M_{0}$ and synthetic IM recorded in presence of one type of misregistration):

$$
\delta I M_{\alpha}=I M_{\alpha}-I M_{0}
$$

with $\alpha$ standing for shift, rotation or magnification.

That way, any $I M^{*}$ can be expressed:

$$
I M^{*}=\alpha_{0} I M_{0}+\alpha_{x} \delta I M_{x}+\alpha_{y} \delta I M_{y}+\alpha_{\text {rot }} \delta I M_{\text {rot }}+\alpha_{m a g n} \delta I M_{\text {magn }}
$$

The orthogonality of the $\delta I M_{\alpha}$ is ensured by the normalization of $I M_{0}$ that gives the scaling factor $\alpha_{0}$. The misregistration parameters $\alpha_{x}, \alpha_{y}, \alpha_{\text {rot }}$ and $\alpha_{\text {magn }}$ can be obtained by projecting the $I M^{*}$ on the sensibility matrix.

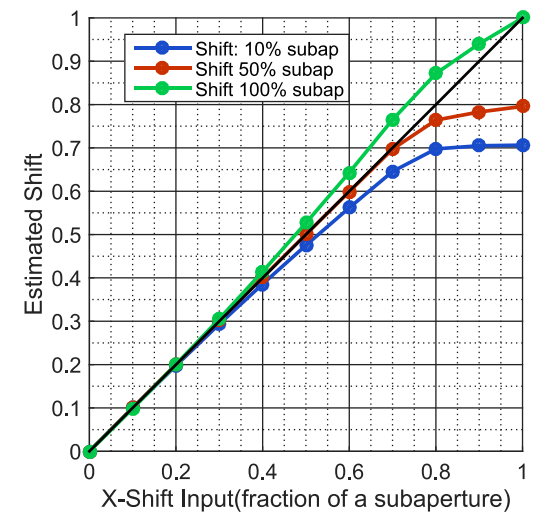

(a) Sensibility of the misregistration identification process

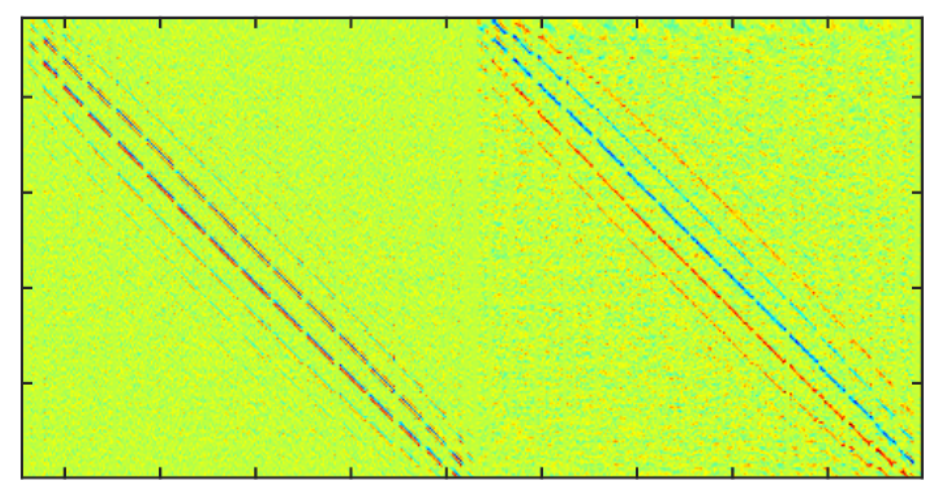

(b) Noisy zonal IM (DM Actuators $\times$ WFS slopes) retrieved from closed-loop data (40 000 frames with $\Delta T=5$ )

Figure 8: Using closed loop data to track the misregistration of the system 
To validate this process of identification and study its sensitivity, first tests have been done by generating 10 synthetic IM (noise-free) with an X shift starting from $0 \%$ to a full subaperture with a step of $10 \%$. These IM are then projected on 3 different $\delta I M$ built with a different shift in X: $10 \%, 50 \%$ and $100 \%$ of a subaperture. The result is displayed in $8 \mathrm{a}$ and shows the linear regime for the identification. In Figure $8 \mathrm{~b}$ is displayed an exemple of Noisy IM that was retrieved using 40000 iterations and a step $\Delta T$ of 5 . The identification of misregistrations from such a noisy IM is still on-going work.

\section{A CALIBRATION STRATEGY FOR THE ELT}

The goal is here to present a first calibration strategy for the ELT, taking in consideration the actual methods studied in this paper to overcome the ELT constraints. The strategy is illustrated in Figure 9.

Without any calibration source, the first IM of the system would be generated synthetically from models and using an M4 like SLM (Spatial Light Modulator DM with a large number of actuators) during the AIT phase. This synthetic IM would be used during the first commissioning night to get a measurement of the IM on-sky that would take into account any difference with the model.

This measurement of the IM would then feed the models of the WFS and DM to generate a first PSIM. The PSIM would be used to start the operations and would be regularly updated using the misregistration identifications provided by the closed loop data. In case of performance drop we could imagine a calibration night during which the IM is acquired on-sky again to update the models and start using a PSIM again.

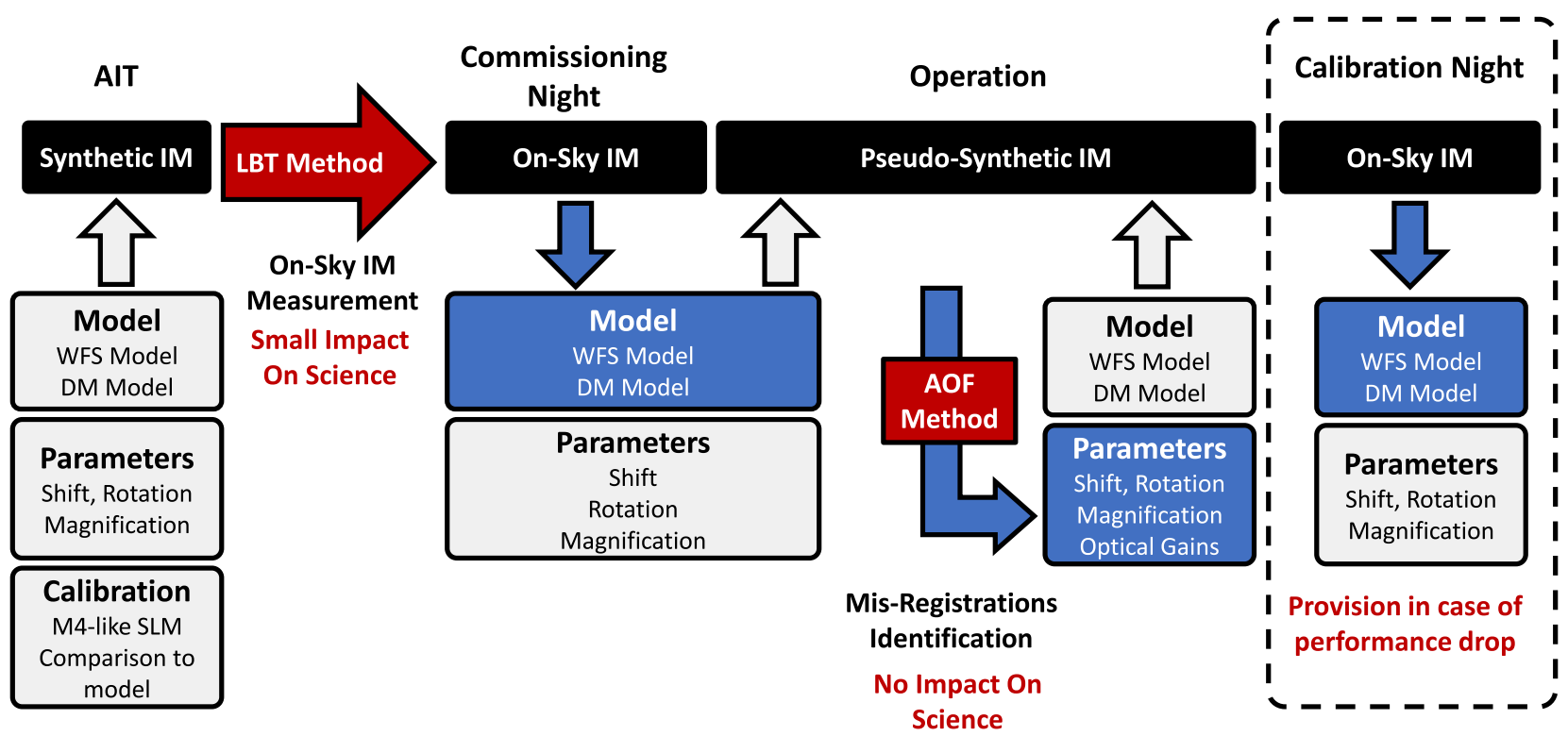

Figure 9: Proposal for a calibration strategy for the ELT

\section{CONCLUSION AND PERSPECTIVES}

In this communication, we have presented the context of the $\mathrm{AO}$ calibration for the first generation of ELT instruments, pointing out the main challenges that need to be overcome to benefit from the full potential of the telescope.

The first method investigated using an on-sky IM has been validated in simulations, and provided interested results even using large misregistration but only using a medium-order AO system. Using it for a high order system with large misregistrations remains to be investigated as it may need a long calibration time. Optimization of the method are still on-going work. 
The second method based on PSIM seems to be ideal for an ELT as it has no impact on science and generate noise-free IM but it relies on complex models with fast and accurate misregistrations identification. Although accurate misregistrations identifications were achieved at the AOF using closed loop data, this wasn't achieved yet using our numerical simulations with Pyramid WFS. Moreover, as the Pyramid WFS model will be extremely complex for the ELT, a thorough study is necessary to reach the precision necessary to use it and generate PSIM. This method remains then to be investigated, also because the amplitude of the misregistrations will be much larger and will evolve regularly with the telescope (based on an ESO internal study for the first generations of instruments of the ELT).

In any case, a fast and accurate identification of the misregistrations is necessary. If this can be done using closed loop data, it will provide an impact-free identification, ideal for a PSIM-based calibration. If not, what could be done is to use the on-sky calibration method of modulation/demodulation process using either specific patterns or modes to identify misregistrations but this remains to be investigated.

\section{ACKNOWLEDGMENTS}

This project has received funding from the European Union's Horizon 2020 research and innovation programme under grant agreement No 730890. This material reflects only the authors views and the Commission is not liable for any use that may be made of the information contained therein.

\section{REFERENCES}

[1] Oberti, S., Quirós-Pacheco, F., Esposito, S., Muradore, R., Arsenault, R., Fedrigo, E., Kasper, M., Kolb, J., Marchetti, E., Riccardi, A., et al., "Large DM AO systems: synthetic IM or calibration on sky?," in [Proc. SPIE], 6272, 627220 (2006).

[2] Kolb, J., Madec, P.-Y., Louarn, M. L., Muller, N., and Béchet, C., "Calibration strategy of the AOF," in [Proc. of SPIE Vol], 8447, 84472D-1 (2012).

[3] Kolb, J., "Review of ao calibrations, or how to best educate your AO system," in [SPIE Astronomical Telescopes+ Instrumentation], 99090K-99090K, International Society for Optics and Photonics (2016).

[4] Pieralli, F., Puglisi, A., Quirós-Pacheco, F., and Esposito, S., "Sinusoidal calibration technique for Large Binocular Telescope system," Adaptive Optics Systems N. Norbert Hubin and EM Claire and and PL Wizinowich and eds 7015, 70153A (2008).

[5] Esposito, S., Tubbs, R., Puglisi, A., Oberti, S., Tozzi, A., Xompero, M., and Zanotti, D., "High SNR measurement of interaction matrix on-sky and in lab," Proc.SPIE 6272, 6272 - $6272-8$ (2006).

[6] Pinna, E., Quirós-Pacheco, F., Riccardi, A., Briguglio, R., Puglisi, A., Busoni, L., Arcidiacono, C., Argomedo, J., Xompero, M., Marchetti, E., et al., "First on-sky calibration of a high order adaptive optics system," in [Proc. SPIE], 8447, 84472B (2012).

[7] Kolb, J., Muller, N., Aller-Carpentier, E., Andrade, P., and Girard, J., "What can be retrieved from adaptive optics real-time data?," in [Proc. of SPIE Vol], 8447, 84475U-1 (2012).

[8] Béchet, C., Kolb, J., Madec, P.-Y., Tallon, M., and Thiébaut, E., "Identification of system misregistrations during ao-corrected observations," (2011).

[9] Béchet, C., Tallon, M., and Thiébaut, E., "Optimization of adaptive optics correction during observations: Algorithms and system parameters identification in closed-loop," in [SPIE Astronomical Telescopes+ Instrumentation], 84472C-84472C, International Society for Optics and Photonics (2012).

[10] Vernet, E., Cayrel, M., Hubin, N., Mueller, M., Biasi, R., Gallieni, D., and Tintori, M., "Specifications and design of the E-ELT M4 adaptive unit," in [Adaptive Optics Systems III], 8447 (2012).

[11] Kasper, M., Fedrigo, E., Looze, D. P., Bonnet, H., Ivanescu, L., and Oberti, S., "Fast calibration of highorder adaptive optics systems," JOSA A 21(6), 1004-1008 (2004).

[12] Meimon, S., Fusco, T., and Petit, C., "An optimized calibration strategy for high order adaptive optics systems: the slope-oriented Hadamard actuation," in [1st AO4ELT conference-Adaptive Optics for Extremely Large Telescopes], 07009, EDP Sciences (2010).

[13] Conan, R., Correia, C., et al., "Object-oriented Matlab adaptive optics toolbox," SPIE (2014).

[14] Roddier, N., "Atmospheric wavefront simulation using Zernike polynomials," Optical Engineering 29(10), 1174-1180 (1990). 